\title{
28 Research Square \\ Hookworm infection in stomach: A case report found by physical examination
}

\section{Di Zhao}

Jilin University First Hospital https://orcid.org/0000-0002-8630-8908

\section{Ronghua He}

Basic Medicine School of Jilin University

\section{Yiqin Wang}

Jilin University First Hospital

Hongyu Jiang ( $\nabla$ jiang_hy@jlu.edu.cn )

Jilin University First Hospital https://orcid.org/0000-0002-3618-5567

\section{Short report}

Keywords: hookworm, stomach, physical examination

Posted Date: May 6th, 2021

DOI: https://doi.org/10.21203/rs.3.rs-155828/v2

License: (9) This work is licensed under a Creative Commons Attribution 4.0 International License. Read Full License 


\section{Abstract}

\section{Background}

Hookworm disease is one of the most widely distributed parasitic diseases in the world. It is endemic in Europe, America, Africa and Asia ${ }^{[1]}$. The main clinical manifestations of hookworm disease are anemia, malnutrition, gastrointestinal dysfunction, and labor decline. It is caused by the parasites of Ancylostoma duodenale Dubini and (or) Necator americanus Stiles. The larvae can invade human capillaries through the skin or mucous membranes, enter the bloodstream, reach the lungs through the right heart, destroy the capillaries of the lungs, enter the alveoli, travel along the bronchi to the pharynx, enter the digestive tract and reach the small intestine after swallowing, and develop into adults in the upper part of the small intestine Attached to the intestinal mucosa ${ }^{[2]}$.

\section{Methods}

We described one case of a businesswomen who sufferd from persistent symptoms of anemia, weight loss, and fatigue for years. Anemia was not significantly improved after routine antianemia treatment. In order to further evaluate the cause of anemia, a gastroscopy was performed, and the microscope showed a single hookworm in the lower stomach. Diagnosis was confirmed by the successful funding of Hookworm eggs in the lining of excrement.

\section{Conclusions}

Hookworm is a human parasite transmitted through the faecal-oral route which reside the duodenal bulb normally ${ }^{[3]}$. Adult worms are not usually seen in the stomach. There are only few cases reporting hookworm infestation of the stomach. In this case report we present an endoscopic demonstration of hookworm infestation in the stomach found by physical examination of a woman who presented with chronic anaemia.

For anaemia patients, especially chronic anaemia patients and those suspected of hookworm disease, gastroscopy should pay attention to the observation of duodenal globules and fecal search for eggs, which are conducive to the early diagnosis of hookworm disease.

\section{Case Presentation}

\section{Background}

Hookworm disease is one of the most widely distributed parasitic diseases in the world. It is endemic in Europe, America, Africa and Asia ${ }^{[1]}$. The main clinical manifestations of hookworm disease are anemia, malnutrition, gastrointestinal dysfunction, and labor decline. It is caused by the parasites of Ancylostoma duodenale Dubini and (or) Necator americanus Stiles. The larvae can invade human capillaries through the skin or mucous membranes, enter the bloodstream, reach the lungs through the right heart, destroy 
the capillaries of the lungs, enter the alveoli, travel along the bronchi to the pharynx, enter the digestive tract and reach the small intestine after swallowing, and develop into adults in the upper part of the small intestine Attached to the intestinal mucosa ${ }^{[2]}$.

\section{Methods And Results}

The patient's feces and the removed parasites were sent to the Parasitology Department for further clarification, and the results suggested that the parasite was clearly diagnosed as hookworms, and hookworm eggs were found in the patient's feces (Figures 2). The patient was definitely diagnosed with gastric hookworm disease. To be treated with antiparasitic drugs (albendazole) and iron supplementation.

\section{OUTCOME AND FOLLOW-UP}

After removing the parasites for 1 week, the patient's hemoglobin improved囚HBG 103g/L囚. After 6

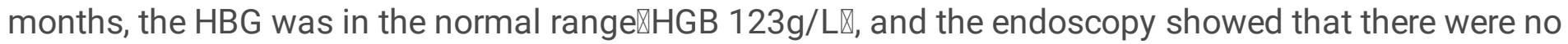
parasites in the stomach, only superficial gastritis.

\section{Discussion}

Hookworm infections are common in the tropics and subtropics. The prevalence of hookworm infection is highest in sub-Saharan Africa, followed by Asia, Latin America, and the Caribbean. It is estimated that over 800 million people are infected with hookworms worldwide ${ }^{[3]}$.The prevalence of hookworm infection in China is $6.12 \%$, with neraly 39.3 million infected people ${ }^{[1]}$.

A diagnosis of hookworm infection includes a history of skin exposure to potentially contaminated soil, clinical manifestations, and stool examination, which is the primary established tool. The patient lives in the river basin, prefer to eat raw fish and seafood, had a history of travel to the southern of china and barefoot walking. Clinical manifestations include Chronic nutritional impairment, pulmonary infection and gastritis. The result of stool egg testing was positive. The final diagnosis of hookworm infection was established.

The hookworm life cycle begins with passage of eggs from an adult host into the stool. Hookworm eggs hatch in the soil to release rhabditiform larvae that mature into infective filariform larvae. Infection is transmitted by larval penetration into human skin; as few as three larvae are sufficient to produce infection ${ }^{[4]}$. From the skin, larvae migrate into the blood vessels and are carried to the lungs. Approximately 8 to 21 days following infection, larvae penetrate into the pulmonary alveoli, ascend the bronchial tree to the pharynx, and are swallowed. In addition to percutaneous larval penetration (the principal mode of transmission), some species of hookworm infection may also be transmitted by the oral route. 
The hookworm are not usually seen in the stomach. So far only a few reports of hookworm infestation of the stomach are available ${ }^{[5]}$. In this case, we didn't seen any parasite in duodenum or other intestinal tract but single one in stomach. The proposed mechanism by which the worm parasite in the stomach body might be relationed to the chronic gastritis and low immunity. Chronic gastritis results in gastric acid secretion change, and Long-term anemia and long medical history leads to low immunity. Provides a condition for hookworm to parasitism in the stomach.

Anemia is the main symptom of hookworm disease. Studies have shown that the mechanisms by which hookworms cause anemia are manifold. Hookworms attach to the mucosa of the gastrointestinal tract through biting, and the blood vessels at the attachment site will leak and lose blood due to the biting of hookworms. In addition, hookworms can cause the secretion of anticoagulants, leading to blood loss. In addition, it was found that hookworm infection can cause iron loss. In the case of moderate to severe hookworm infection, when the blood loss exceeds the host's intake and iron and protein reserves, chronic IDA will occur.

This patient has multiple cysts in liver and kidney in CT, round saccate shadows were seen in the splenic hilum with marginal calcification. Multiple round low-density shadows were seen locally in the right lung, subpleural nodules were in the upper lobe of both lungs. Whether the above changes are related to hookworm infection remains to be further observed.

\section{Conclusion}

Hookworms have been infecting humans for a long time, spreading across the world, and seriously endangering human health after infection. Due to their lack of specific symptoms and signs, they are easily missed and misdiagnosed. This case may indicate that the patient is clinically found to have symptoms of anemia or gastrointestinal bleeding, but it is difficult to find the cause of the cause, consider whether there is the possibility of hookworm infection, and perform endoscopy and fecal worm egg detection as soon as possible. Early diagnosis and treatment will reduce The continuous damage of hookworms to the human body.

\section{Declarations}

\section{Ethical approval}

Wrritten informed consents were obtained from the patients for publication of this case report.

\section{Competing interests}

The authors declare that they have no competing interests.

\section{Authors' contributions}


JHY designed the study, supervised drafted the manuscript, ZD contributed to case presentation and drafted the manuscrip, HRH porformed fecal egg detection, WYQ participated in drafted the manuscript. All authors read and approved the final version of the manuscript.

\section{Author details}

${ }^{1}$ Department of physical examination center, The $1^{\text {st }}$ Hospital of JiLin University, Xinmin Street 1, JiLin, China. ${ }^{2}$ Department of Former Biology Teaching and Research Office, Basic Medical College of Jilin University, Xinmin Street 126, JiLin, China.

\section{Competing interests}

The authors declare that they have no competing interests.

\section{Funding}

Jilin Science and Technology Development Plan Program (20190902012TC), Jilin Science and Technology Development Plan Program (20190304129YY).

\section{References}

[1]Dongxing Zhang,Fangwei Wu. Progress in prevention and treatment of hookwworm disease in China[J].China Tropical Medicine,2019,19(2):188-191.

[2]Lanjuan Li,Hong Ren. Infectious Diseases[M].BeiJing:People's Medical Publishing House,2013,322-325.

[3]Simon Brooker, Jeffrey Bethony, Peter J Hotez. Human hookworm infection in the 21st century. Adv Parasitol. 2004;58:197-288. doi: 10.1016/S0065-308X(04)58004-1.

[4]Roche M, Layrisse M. The nature and causes of "hookworm anemia". Am J Trop Med Hyg 1966;15:1029-102.

[5]Boopathy V, et al. Endoscopic (video) demonstration of hookworm infestation of the stomach. BMJ Case Rep 2014. doi:10.1136/bcr-2014-204065.

\section{Figures}




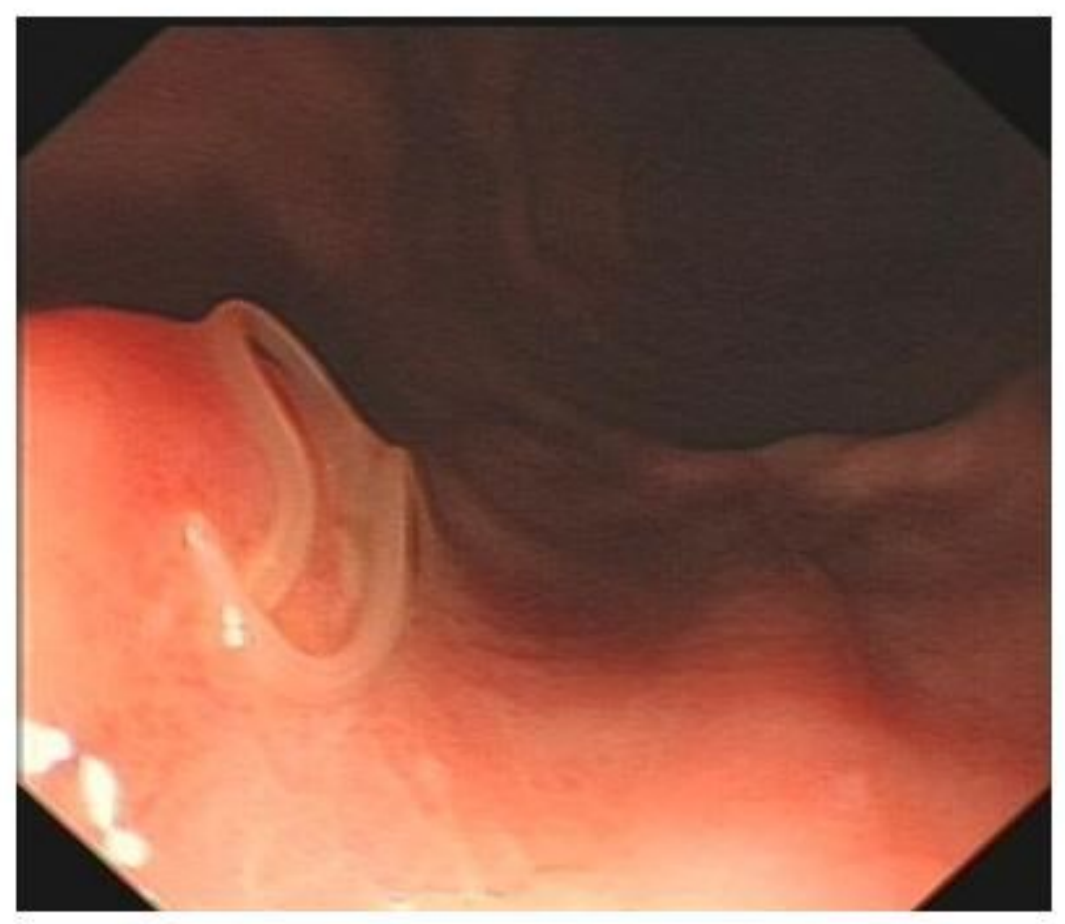

Figure 1

Hookworm adsorption on the stomach

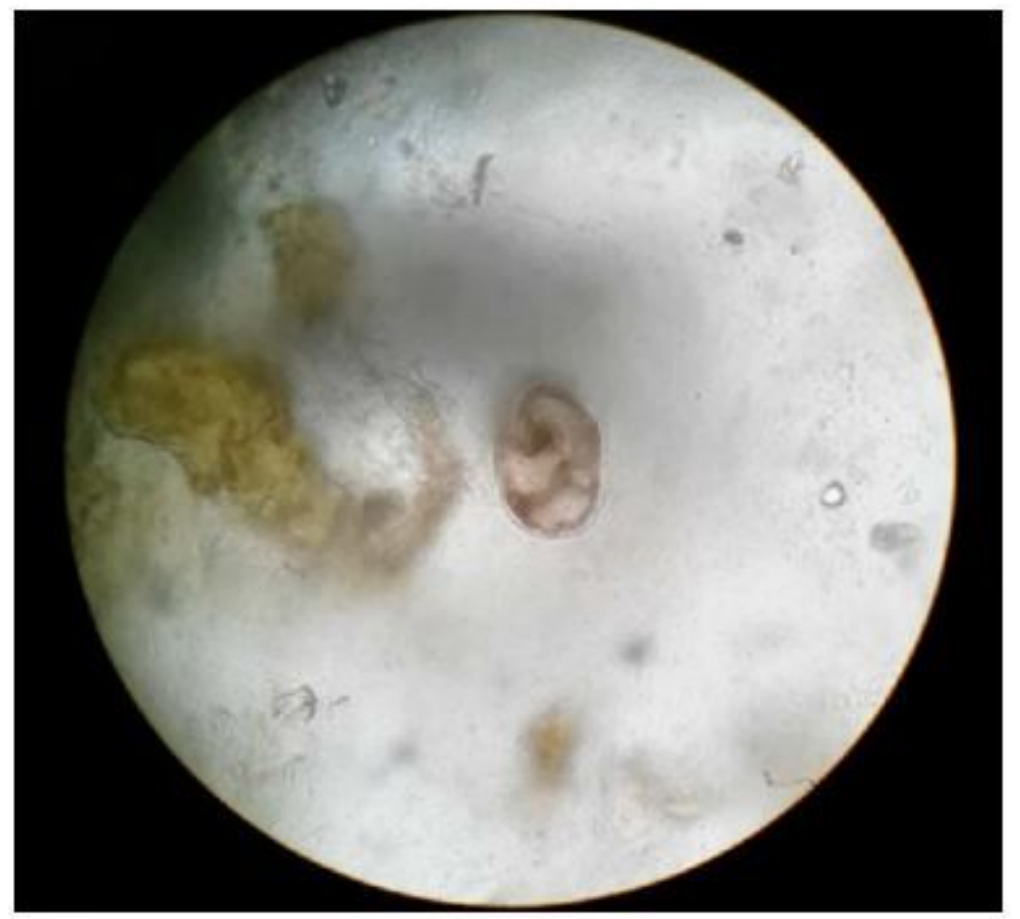

Figure 2

Hook worm eggs in the lining of excrement 